\title{
Kartagener's Syndrome: A Case Report in the Samutsakhon Hospital
}

\author{
Luckana Girapongsa', Thanapat Puangpetch², \\ Orawan Autravisitkul', Pariyanoot Deesuwun' \\ ${ }^{1}$ Department of Radiology, Samutsakhon Hospital, Thailand. \\ ${ }^{2}$ Department of Medicine, Samutsakhon Hospital, Samutsakhon, Thailand.
}

\begin{abstract}
Kartagener's syndrome is an autosomal recessive disorder. ${ }^{1}$ It is characterized by the triad of situs inversus, bronchiectasis and sinusitis. Basic problem is defective movement of the cilia. ${ }^{3}$ Recognition in this condition keeps prevention for unnecessary investigation. We have presented a case of "Kartagener's syndrome" in the Samutsakhon hospital.
\end{abstract}

Keyword: Kartagener's syndrome, primary ciliary dyskinesia, situs inversus. 


\section{Introduction}

Kartagener's syndrome is a rare autosomal recessive disorder syndrome with an estimated incidence about 1: 20,000-30,000. ${ }^{2}$ Male and female are effected equally. ${ }^{6,11}$

Manes Kartagener, a pulmonologist in Zurich , described an unsual triad of situs inversus, bronchiectasis and sinusitis in four patients and became known as Kartagener's syndrome in 1933. ${ }^{8}$ Actually, it was firstly described in literature by Sievert in 1904 who reported the case of a 21-years-old man in whom the three elements of syndrome were presented. ${ }^{6}$ Our case was diagnosed as Kartagener's syndrome.

\section{Case report}

History

A 29 -years-old female presented with dyspnea for 3 hours. She asked for emergency medical service for taking her from home to the Samutsakorn hospital. Her past medical history showed that she was used to be treated as pulmonary TB from another hospital since many years.

The vital sign; T $36.5^{\circ} \mathrm{C}$, PR 140/ min, RR 40 / $\mathrm{min}, \mathrm{BP} 109 / 44 \mathrm{mmHg}$. The physical examination revealed crepitation and rhonchi at both lungs. The other physical examinations are within normal limit.

The CBC; Hemoglobin $9.7 \mathrm{~g} / \mathrm{dl}$ (anisocytosis 1+ and ovalocyte 1+), WBC 15,800 cell / uL (neu-

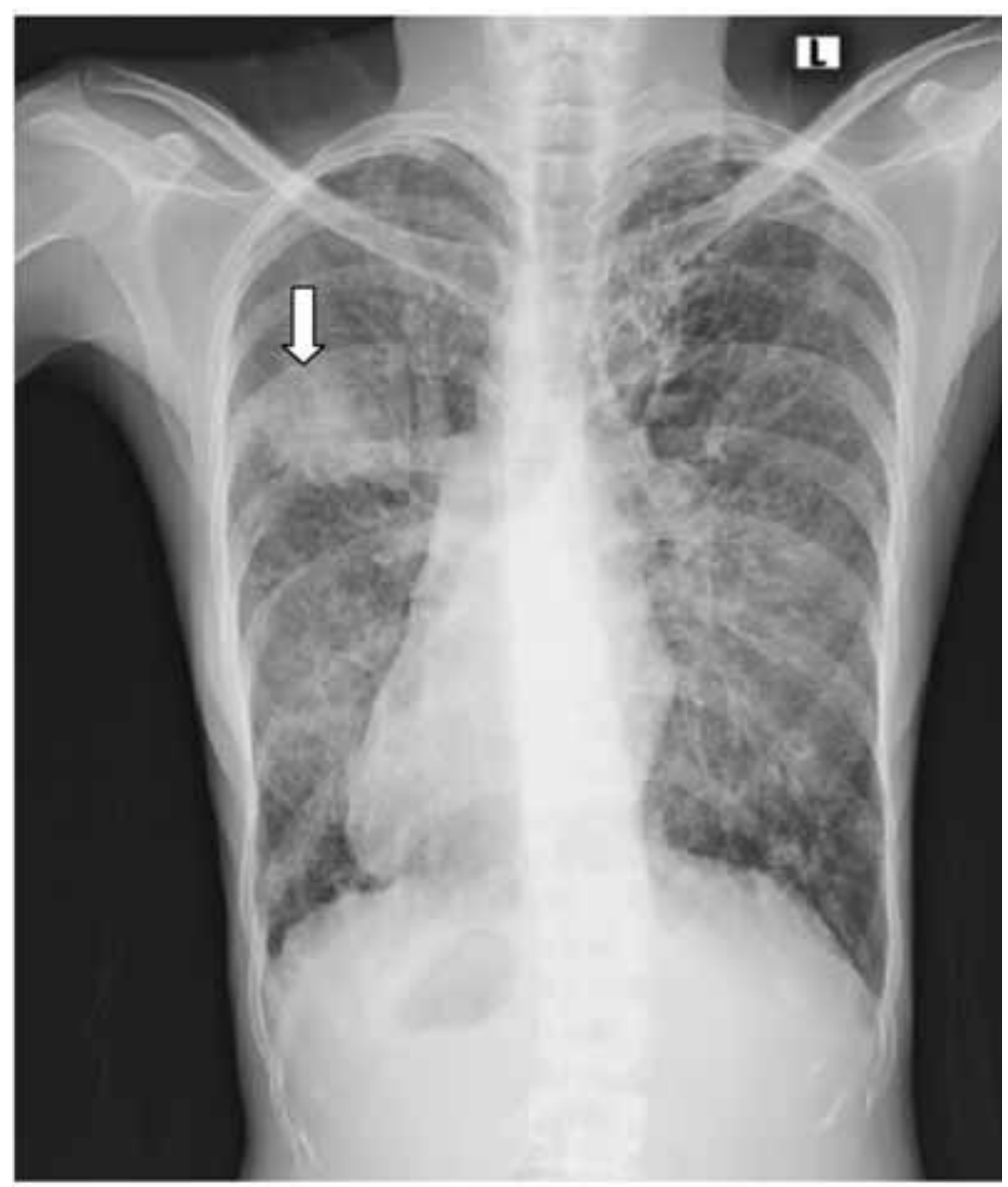

Figure 1. The chest radiography revealed alveolar infiltration at RUL (arrow). Bilateral bronchiectasis and dextro-cardia were seen. 


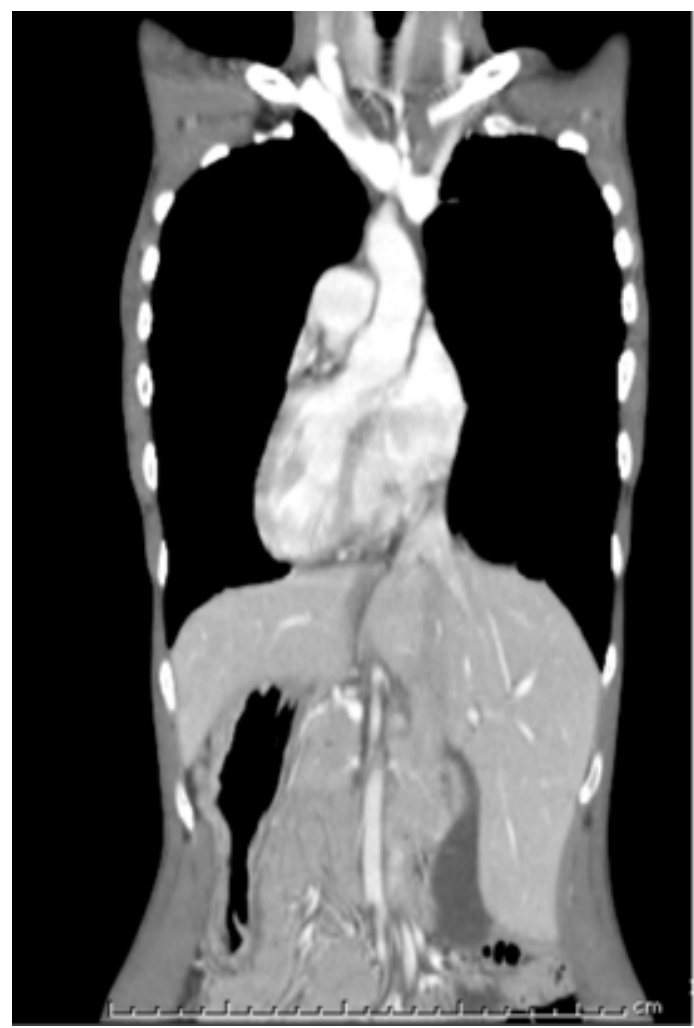

Figure 2. CT of the chest (coronal view), there were left position of the liver and right position of the spleen / stomach. Dextrocardia was noted.

trophil $89.3 \%$, lymphocyte $7.9 \%$, monocyte $2.1 \%$ ) and platelet count 753,000 cell/ UL. His laboratory investigation including kidney and liver function test were within normal limited.

She performed the radiography of the chest. The chest study revealed alveolar infiltration at RUL with diffuse bronchiectasis at both lungs. Right side of the cardiac apex is noted.

Then she performed the computed tomography of the chest. The computed tomographic findings revealed right position of the cardiac apex / the anatomic left atrium / left ventricle, the rightsided of the aortic knob including descending aorta, the right upper quadrant position of the stomach / spleen, the left upper quadrant position of the liver / gallbladder, left -sided trilobed lung and right-sided bilobed lung which were mirror-image to situs solitus representing situs inversus totalis.
There were multi-focal alveolar infiltrations with nodules at the apico-posterior segment of RUL, the superior segment / the anterior basal segment / lateral basal segment of RLL with some tree-in bud appearance; representing multi-focal pneumonia. There were cystic bronchiectasis with partial atelectasis at LUL and the medial basal segment of LLL.

Additional radiography of the para-nasal sinuses was also performed. The study revealed opacification of the right maxillary sinus and mucoperiosteal thickening of the left maxillary sinus representing sinusitis.

Thus, Kartagener's syndrome was diagnosed clinically. She was admitted ad treated with intravenous antibiotics and bronchodilators. The majority of symptoms improved and was discharged with oral antibiotics. 


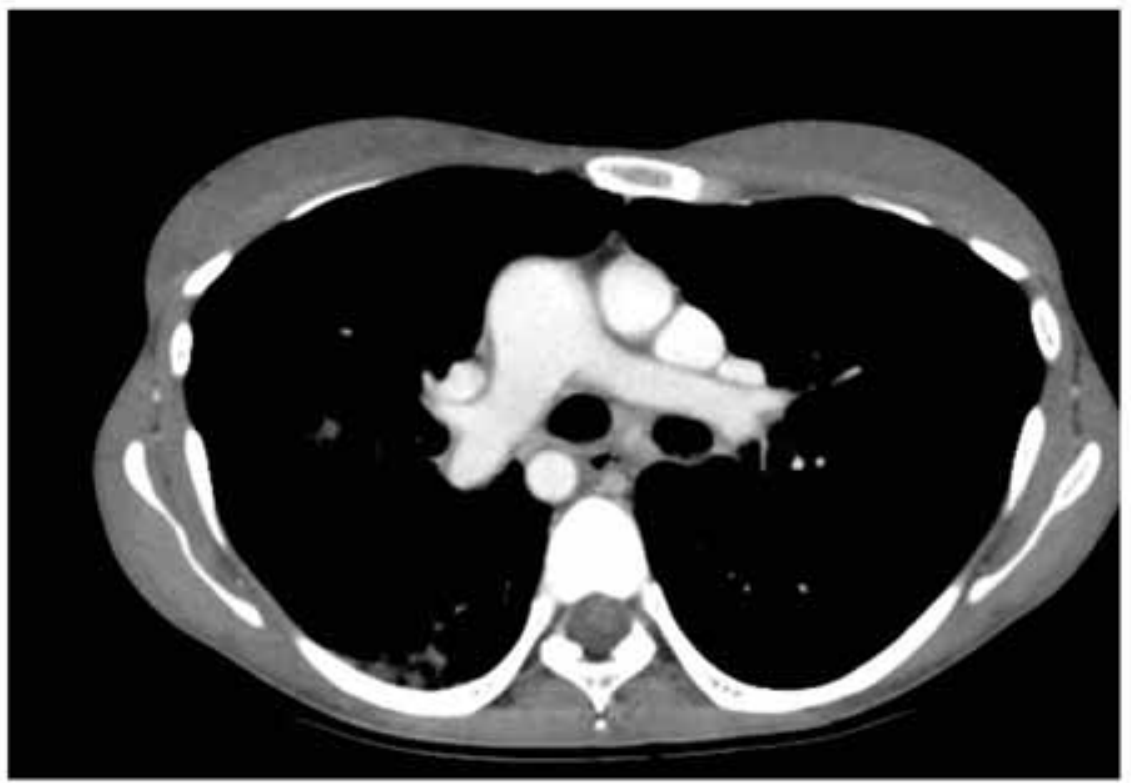

Figure 3. CT of the chest (axial view), there were alveolar infiltration at posterior segment of RUL and the superior segment of RLL. Right side aortic arch and descending aorta were noted.

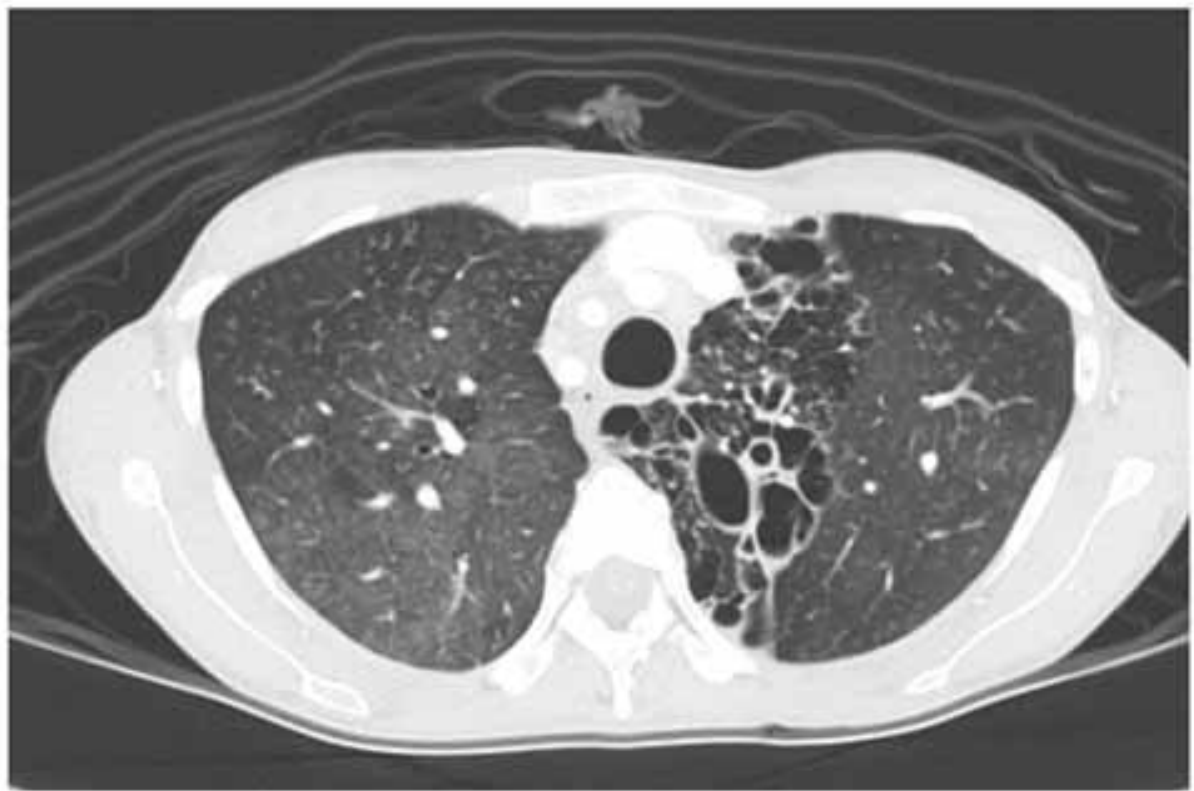

Figure 4. CT of the chest (axial view and lung window) There were cystic bronchiectasis at LUL. 

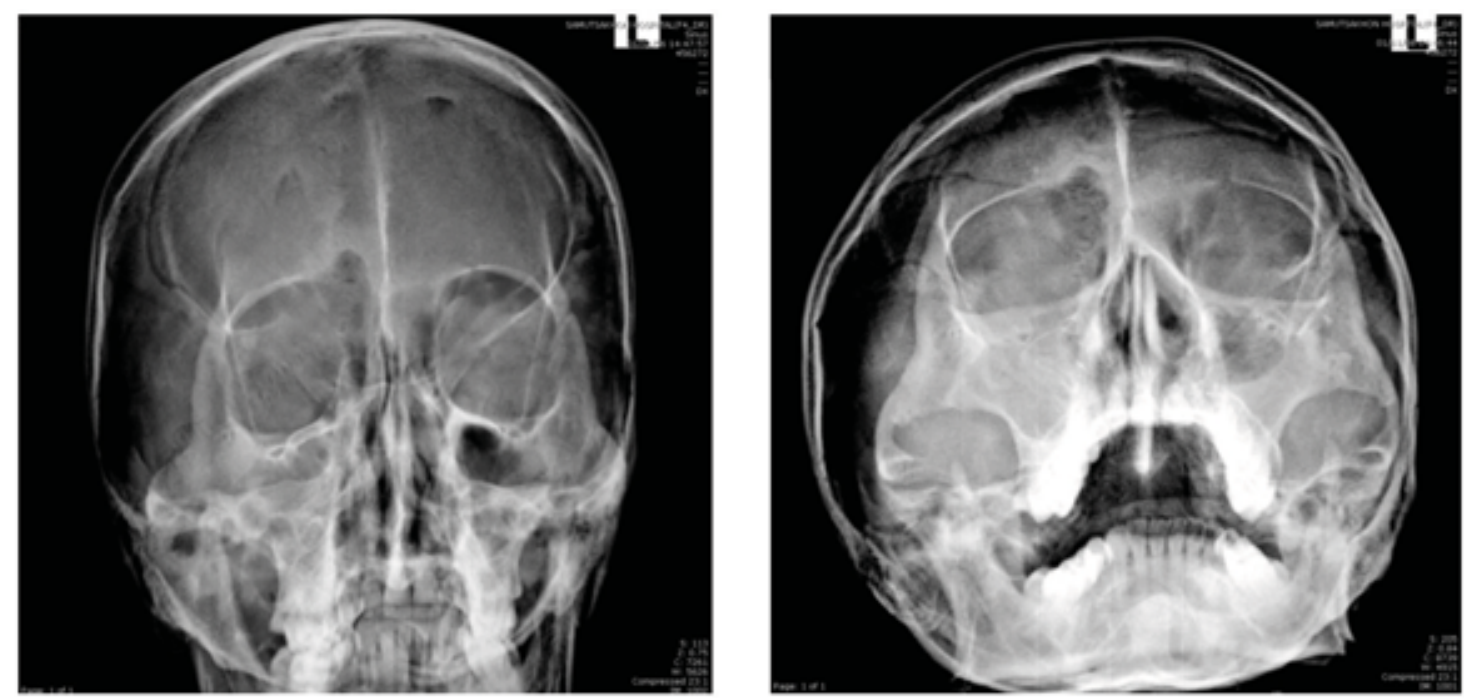

Figure 5. The paranasal sinuses study were opacification of the right maxillary sinus and mucoperiosteal thickening of the left maxillary sinus.

\section{Discussion}

Kartagener's syndrome is recognized as the clinical variant of the primary ciliary dyskinesia (PCD) 5 and occurs about $50 \%$ of PCD. The primary ciliary dyskinesia is autosomal recessive disorder characterized by inefficient or absent mucociliary clearance. $^{5}$

The pathology is based on the ultra structural defects of the cilia. 5 Cilia can be structurally divided into subcomponents that include a basal body, transitional zone, axoneme, ciliary membrane and ciliary tip. ${ }^{10}$ Cilia can either as motile (in air ways, brain, fallopian tube, sperm, embryonic node) or nonmotile (in kidney tubule, bile duct, pancreatic duct, eye, ear, bone cartilage and fibroblast). ${ }^{10}$ Normally in most motile cilia, the core or axoneme of the cilia consists of a 9+2 microtubule structure with a ring of nine microtubules doublets surrounding a central pair of single microtubule. The nine microtubule doublets are studded with dynein arms that contain adenosine triphosphatase and act as molecular motor to effect the sliding of the peripheral micro- tubule pairs relative to one another. ${ }^{2,5}$ The $9+0$ axonemes that are found in most of non-motile cilia lack the two central microtubules and lack of dynein arms. $^{10}$

Most of the disease results from mutations indentified to date involve two genes such as genes encoding for the dynein axonal intermediate chain 1 (DNAI1) and the dynein axonemal heavy chain 5 (DNAH 5). ${ }^{5}$ Since dynein is one of key intracellular molecular motors, the absence of dynein arms is responsible for the impaired molitity of the cilia and sperm. $^{7}$

The clinical features depend on the involved systems. In the upper respiratory tract, rhino-sinusitis and otitis media are cardinal features of disease and responsible for much of the morbidity associated with PCD in early childhood. Nasal congestion and rhinorhea are very common. ${ }^{5}$ In the lower respiratory tract, most patients report a chronic and productive cough as a prominent symptom because cough compensates for lack of effective mucociliary clearance and impaired muco-ciliary clearance 
lead to recurrent episode of pneumonia or bronchitis. ${ }^{5}$ Bronchiectasis does not present at birth but may develop early sometimes as early as in childhood. ${ }^{2}$ Bronchiectasis is an acquired condition. Involved bronchi are dilated, inflamed and easily collapsible resulting in airflow obstruction and impaired clearance of secretion. Insufficient clearance of secretion causes colonization and infection with pathogen organisms contributing to common purulent expectoration in patients with bronchiectasis resulting in further bronchial injury and viscous cycle of bronchial damange, bronchial dilatation, impaired clearance of secretions, recurrent infection and more bronchial damage. ${ }^{6}$ The most common respiratory pathogens are Haeophillus influenza, Streptococcus pneumonia. ${ }^{4}$

Laterality defects, cilia on the embryonic node play critical role in left- right patterning during early development. ${ }^{5}$ Situs inversus totallis, heterotaxy with or without congenital cardiovascular abnormality are observed. In other organ, such as in the genitourinary tract, male fertility is common and reflects defects in sperm tail axonems. In the central nervous systems, hypothetically due to impaired CSF flow secondary to dysfunction motile cilia that line the ventricular ependymal cells may be linked with hydrocephalus. In the eye, some may develop with retinitis pigmentosa. ${ }^{5}$

Diagnosis of Kartagener's syndrome is usually achieved by clinical features and radiological images. ${ }^{6}$ Other investigations are also helpful such as quantitative decreased in number of dynein arm and subjective abnormality in other ciliary compents on electrom microscopy. ${ }^{9}$

Treatment is aimed to relieved symptoms and prevents complications. Early recognition of the disease and prompt antibiotic treatment are the key to minimize the irreversible lung damage. Physiotherapy with postural drainage and cessation of smoking are also important. ${ }^{4}$ Coughing should not be suppressed since it acts as a substitute for mucociliary clearance. ${ }^{4}$ Prophylactic measures such as appropriate immunization particular influenza vaccines and pneumococcal vaccine are mainstay. ${ }^{6}$

The prognosis is generally considered favorable and life expectancy is usually normal. ${ }^{6.8}$ There will have progressively greater impact in health in the second half of life producing significant morbidity and restriction of life style. But there is a clinical case demonstrated a non-progressive course of the bronchiectasis indicates that is a complex interrelationship between genetic variation and an appropriate non-specific treatment. ${ }^{8}$ Early diagnosis and hence earlier treatment may improve symptoms and the impact of the condition. ${ }^{\text {? }}$

\section{Conclusion}

Kartagener's syndrome is the clinical variant of the primary ciliary dyskinesia (PCD) and consists of situs inversus, bronchiectsis and sinusitis. Basic pathology is defect of the cilia causing impair mucociliary clearance. Prognosis is good. Early recognition in this disease can improve symptoms and may prevent progressive course.

\section{References}

1. Kartagener M, Horlancher A, Situs vuscerum inversus and polyposisnais in enieum Falle familliaerer Brouchiectasin . Beitr Klin Tub 1936;87:331-3.

2. Bavaliya M, Sadatia V, Vekariya R, Jani D. Kartagener's syndrome. A Rare Case. NJIRM 2012;3(4):139-41.

3. Mahsud I, Din S. Kartagener 's syndrome. Glomal Journal of Medical Science 2006;4(2):79-81.

4. Dabni AS, Chaudihri SR, Thorat PB, Pandya HB, Shah MC, Meswani UN, Vaghela PR. Kartagener's syndrome: 
May-August 2013, Volume XIX No.II

A Triad of Bronchiectasis, Situs inversus and Chronic Sinusitis . JIACM 2005;6(3):241-3.

5. Masumi Akita. Primary Ciliary Dyskinesia/Kartagener Syndrome - Clinical and Genetic Aspects. Approaches to Bronchitis, 2011. (cited 2014 Jan 20) InTech, Available from:http://www.intechopen.com/books/approaches-tobronchitis/primary-ciliary-dyskinesia-kartagener syndromeclinical-and- genetic- aspect.

6. Casonova MS, Tuji FM, Yoo HJ, Haiter-Neto F. Kartagener 's syndrome. Dentmaxillofac Radiology 2006;35(5): 386-9.

7. McManus IC, Mitchison HM, Chung EM, Stubbing GF, Martin N. Primary ciliary dyskinesia (Siewert's / Kartagener's syndrome): BMC pulmonary medicine 2003;3:4.
8. Serapinas D, Staikuniene J, Barkauskiene D, Jackute J, Sakalauskas R. An unusal regression of the symptoms of Kartagener's syndrome. Arch Bronconeumol 2013;49(1): 28-30.

9. Teknos TN, Metson R, Chasse T, Belecia G, Dickersin GR. New development in the diagnosis of Kartagener's syndrome. Otolaryngol Head Neck Surg 1977;116(1): 68-74.

10. Fliegauf $M$, Benzing $T$, Omran $H$. When cilia go bad: cilia defects and ciliopathies. Nat Rev Mol cell Bio 2007; 8(11):880-91.

11. Homma S, Kawabata M, Kishi K, T suboi E, Narui K, Saiki S etal. Bronchiolitis in Kartagener 's syndrome. Eur Respir J 1999:14:1332-9. 